\title{
Research Square \\ Determinants of Clinician and Patient to prescription of antimicrobials: case of Mulanje, Southern Malawi
}

Morris Chalusa ( $\sim$ morrischalusa@yahoo.com )

Ministry of Health https://orcid.org/0000-0001-9451-3808

Felix Khuluza

University of Malawi College of Medicine

Chiwoza Bandawe

University of Malawi College of Medicine

\section{Research}

Keywords: Antimicrobial resistance, Antibiotics, Clinician, Patient, Prescription

Posted Date: November 9th, 2021

DOI: https://doi.org/10.21203/rs.3.rs-1044393/v1

License: (c) (i) This work is licensed under a Creative Commons Attribution 4.0 International License.

Read Full License 
1 Determinants of Clinician and Patient to prescription of antimicrobials: case of Mulanje,

2 Southern Malawi

3 Morris Chalusa $^{1,2^{*}}$, Felix Khuluza ${ }^{3}$, Chiwoza Bandawe ${ }^{4}$

$4 \quad{ }^{1}$ Pathology Department, College of Medicine, University of Malawi, Blantyre, Malawi.

$5 \quad{ }^{2}$ Mulanje District Hospital, Ministry of Health, Malawi.

$6{ }^{3}$ Pharmacy Department, College of Medicine, University of Malawi, Blantyre, Malawi.

$7 \quad{ }^{4}$ Department of Mental Health, College of Medicine, University of Malawi, Blantyre, Malawi.

$8 \quad$ *Corresponding author: morrischalusa@yahoo.com

9 Pathology Department, College of Medicine, University of Malawi, Blantyre, Malawi.

10 Mulanje District Hospital, Ministry of Health, Malawi 
20 Background: Antimicrobial resistance is an emerging problem in low- and middle-income 21 countries. The problem is exacerbated by inappropriate prescription of antimicrobials. Factors 22 leading to overuse or inappropriate prescription of antimicrobials by the cadre of medical 23 assistants, clinical technicians and clinical officers have received limited attention. This study 24 investigated factors that influence prescription behaviours of antimicrobials among clinical 25 officers in various health facilities in Mulanje district, Southern Malawi.

26 Methods: In-depth interviews $(n=18)$ and focus group discussions $(n=2)$ were conducted with

27 COs from four health facilities in Mulanje district. Purposive sampling was done to arrive at a 28 sample size of 30 health cadres.

29 Results: Participants pointed out that patient preferences, belief and clinicians' inadequate 30 education on this issue were among the factors that contribute to inappropriate antimicrobial 31 prescription. $75-\%$ of clinicians showed lack of knowledge on the definition of antibiotic and 32 antimicrobial resistance.

33 Conclusion: Inappropriate use of antimicrobials is facilitated by prescribing decisions made by 34 clinicians who are greatly influenced by their patients. Interventions aimed at improving 35 antimicrobial prescription should target both clinicians and patients.

36 Key words: Antimicrobial resistance, Antibiotics, Clinician, Patient, Prescription. 


\section{Introduction}

39 Antimicrobial resistance (AMR) is a great public health challenge which is accelerated by

40 inappropriate use of antimicrobials [1]. Overprescribing of antimicrobials is associated with

41 increased risks of prolonged hospital stay, self-medication of self-limiting conditions, amplified

42 frequent admission to hospital and causing severe infections [2]. Globally, at least 700,000

43 people die each year of drug resistance illnesses because of infections such as bacteria, malaria,

44 Tuberculosis and HIV / AIDS [3]. A study conducted in Malawi reported that there is a decrease

45 in bacterial bloodstream infection which has been accompanied by a rise in antimicrobial

46 resistance involving all bacterial bloodstream infection pathogens [4]. A recent study done in

47 Malawi found out that Gram-positive pathogens are resistant to empiric, first-line antimicrobials

$48[5]$.

49 Patient-pressure and customer-satisfaction are considered to be major factors for inappropriate 50 antibiotic prescription [6]. In hospital settings, the cadre of Clinicians also known as Clinical

51 Officers (COs) is tasked with prescription of medicines to patients. COs are licensed medical

52 practitioners with an initial three-year training and one year internship [7]. According to

53 Mangione (2019), clinicians are more likely to prescribe antibiotics if they perceive that parents

54 who have brought sick children to the hospital want antibiotics if they ask about the treatment 55 plan [6].

56 Much of the global overuse of antimicrobials occurs in low - and middle-income countries

57 (LMICs), topped by the BRICS (Brazil, Russia, India, China and South Africa) nations where 58 there is enormous use in both animal and human sectors [8]. A study conducted in Sudan, 59 Guinea-Bissau, Central African Republic and Democratic Republic of Congo found out that 60 patient demand for antibiotics contributed to antibiotics prescription [9]. 
61 In Sub-Saharan Africa, one factor contributing to AMR is misuse of antibiotics. However,

62 improvements in malaria diagnostics and the recognition that malaria transmission is decreasing

63 globally have highlighted the lack of tests for other infections and many patients who test

64 negative for malaria are treated with antibiotics indiscriminately [10]. This is where the focus on

65 prescribing habits of clinicians has to be looked at as it may inadvertently contribute to AMR.

66 Some of the barriers to prudent prescribing of antibiotics by general practitioners (GPs) are

67 known, and these are: patients demanding antibiotics, prescribing antibiotics to save time due to

68 the perception that it takes longer to explain why antibiotics are not needed, concerns that the

69 patient may not return for follow up, uncertainty in the diagnosis where antibiotics may be

70 warranted, concerns about possible complications, preservation of the doctor-patient relationship,

71 and knowledge and attitudes to AMR [11].

72 Szymczak explained that clinicians identify patient pressure and demand for antibiotics as a 73 major barrier to more judicious prescribing [12]. Brookes-Howell, et al. described how clinicians

74 spoke of familiarity with the patient, which helped clinicians in their decision on whether or not

75 to prescribe antibiotics [13]. Patient pressure and expectation were cited among the reasons for

76 prescribing antibiotics 'unnecessarily', in particular where a shortage of consulting time meant

77 that the doctor felt unable to adequately explain why antibiotics were inappropriate [14].

78 Physicians' express desire for a quick fix, the problem of diagnostic uncertainty and the fact that 79 explaining why antibiotics are not necessary is too time-consuming and unrewarding [15] were 80 reported as being the basis of antibiotic misuse. Patients' lack of knowledge also drives their 81 demands for antimicrobials [16]. 
82 Doctor's communication skills are the core clinical skills in the practice of medicine, with the

83 ultimate goal of achieving the best outcome and patient's satisfaction which are essential for the

84 effective delivery of health care [17].

85 Communication skills and diagnostic uncertainty rank among the principal indirect factors

86 influencing antibiotic prescription [15]. In a study conducted in Malaysia, the majority of the

87 respondents agreed that too many antibiotic prescriptions, using too many broad spectrum

88 antibiotics and excessive use of antibiotics in livestock were leading contributors to AMR. In the

89 same study, another group felt that too long durations of antibiotic treatment, too low dosing of

90 antibiotics, poor hand hygiene and not removing the focus of infection are among the major

91 factors contributing to AMR [18].

92 However, improving clinician's communication skills helps to bridge the gap between

93 physician's and patient's expectations [19].

94 Antimicrobial prescription can also be reduced in settings where there is one-on-one patient-

95 directed education in the workplace [20].

96 The present paper was aimed at identifying determinants of decisions in antimicrobial

97 prescription among clinicians in health care settings. Since a previous study on antimicrobial

98 prescription focused on physicians and Medical doctors, it was necessary to also get views from

99 Clinical Officers, Clinical Technicians and Medical Assistants, particularly in Sub Saharan

100 Africa who are at the frontline in providing health services in primary and secondary health care

101 settings [21].

102 Methods

103 Study Design, Sample size and Recruitment of participants 
104 This was a qualitative study aimed at exploring clinicians' views and experiences about 105 prescribing AMRs. The research question we tackled was: 'What factors determine the 106 Malawian clinician's decision to inappropriately prescribe Antimicrobials?'. We used in-depth 107 interviews and focus group discussions with clinicians working at the district hospital and health 108 centers in Mulanje district, Southern Malawi. In-depth interviews and focus group discussions 109 were chosen because they provide much more detailed information and they allow for a more 110 relaxed atmosphere. They were also chosen because responses can be clarified and expanded 111 upon with probing question and interviewees can react and build upon each other's response to 112 provide information or ideas that, on their own individually, they might not have provided.

113 Clinicians who completed their internship were selected for inclusion. We recruited clinicians 114 from two hospitals, and two health centers. There were 30 clinicians who took part in the study. 115 Purposive sampling was utilised. This is appropriate in qualitative research, where the aim is not 116 to obtain a statistically representative sample and make statistical inferences from the results, but 117 rather to obtain an information rich sample and make logical inferences from that sample [22]. 118 Recruited clinicians were given a consent form to read and sign, once they had agreed to take 119 part in the study.

120 We collected data between May and June 2019. Clinicians' qualifications ranged from 121 Certificate, Diploma to Degree in Clinical Medicine. All participants were assured of privacy 122 and confidentiality, thus, only the team that was involved in data collection had access to the 123 information. Codes were used instead of names. Ethical approval was sought from Malawi 124 College of Medicine Research Ethics Committee and granted with approval number 125 P.04/19/2656. Permission to collect data in the study health facilities was provided by Mulanje 
District and Mulanje Mission Hospital Directors respectively. Prior to recruitment of clinicians, communication about the study was sent to the health facility Officers-in-charge.

\section{Data collection}

129 Before beginning data collection, we created a semi structured, open - ended interview guide 130 based on literature review on factors that promote inappropriate antimicrobial prescription and 131 piloted with two practicing clinicians. The piloting data was not included in the analysis [23,24].

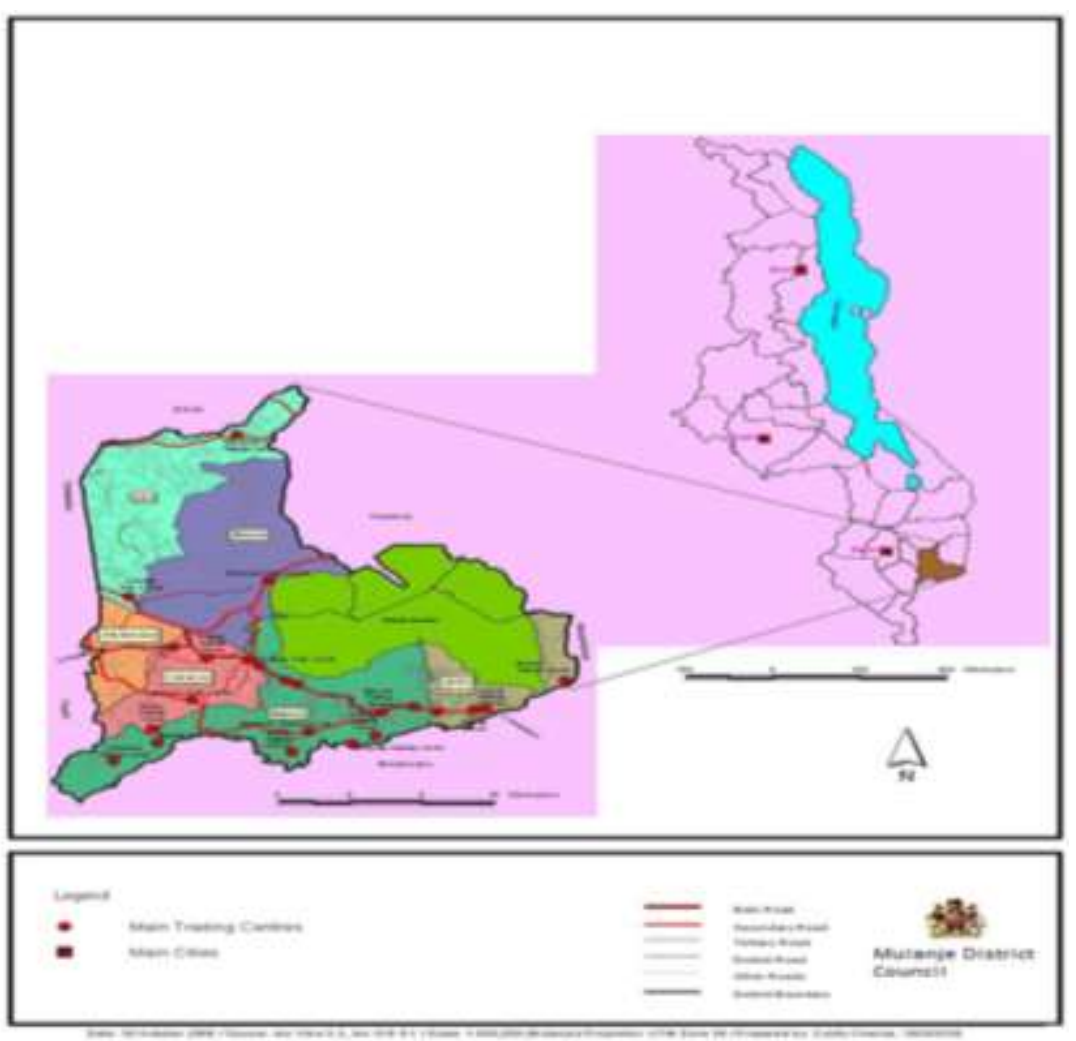

\section{Data analysis}

134 Transcripts were coded using a blend of deductive (codebook based on main interview questions) and inductive coding (emerging from the data). All English transcripts $(n=30)$ were thematically analysed by the primary investigator (MC) and then an independent (CB) researcher

137 with expertise in qualitative research. Analysis began soon after data collection to get 138 familiarization, which involved reading the transcripts repeatedly and noting down ideas. 
139 The information which is pertinent to participants' determinants of antimicrobial prescriptions

140 was identified and coded based on a deductive and inductive approach. These codes were

141 collected into sub themes and themes. The first author subsequently discussed the coding, sub-

142 themes and themes with two independent researchers to enhance data reliability. Themes were

143 reviewed by co-authors (CB and FK). Discrepancies were resolved by reaching a consensus.

144 The first author then presented the findings to the study participants and obtained their feedback

145 to ensure that their perspectives were accurately and clearly represented.

146 Results

\section{Participants' demographic characteristics}

148 There were 17 male and 13 female participants. Their educational backgrounds ranged from

149 Medical Assistant (Certificate in Clinical Medicine), Clinical Technician (Diploma in Clinical

150 Medicine) and Clinical Officer (Degree in Clinical Medicine). All participants $(\mathrm{n}=30)$ reported

151 that they had prescribed antimicrobials in the previous year.

\begin{tabular}{|c|c|c|c|c|c|}
\hline & & $\begin{array}{l}\text { Medical assistant } \\
(\mathrm{n}=11)\end{array}$ & $\begin{array}{l}\text { Clinical Technicians } \\
(\mathrm{n}=16)\end{array}$ & Clinical Officer (=3) & $\begin{array}{l}\text { Overal total } \\
(\mathbf{n}=30)\end{array}$ \\
\hline & Male & 4 & 12 & 1 & 17 \\
\hline Sex & Female & 7 & 4 & 2 & 13 \\
\hline \multirow[t]{2}{*}{ Type of facility } & MMH & & 7 & 2 & 9 \\
\hline & MJDH & 10 & 10 & 1 & 21 \\
\hline Professional qualification & & 10 & 16 & 4 & 30 \\
\hline \multirow{2}{*}{$\begin{array}{l}\text { Professional experience } \\
\text { (years) }\end{array}$} & $<1$ & 5 & 2 & & 7 \\
\hline & $>1$ & 6 & 13 & 4 & 23 \\
\hline
\end{tabular}

152 Main themes

153 Seven main themes influencing antimicrobial prescribing emerged from the semi-structured

154 interviews. Quotations from the interviews are included where relevant to illustrate a point.

155 Theme 1: Patients' Preferences 
156 To explore why clinicians give over their prescribing decision power to patients, the interviewer 157 asked them about determinants of antimicrobial prescription in health care settings. Most 158 clinicians mentioned that some patients force clinicians to prescribe antimicrobials while other 159 patients come with their own diagnosis to the hospital. The clinicians added that a patient's signs and symptoms and a patient's preference for antimicrobials are significant determinants of

161 antimicrobial prescriptions. Most clinicians reported that patients preference on antimicrobials was a factor that contributed to inappropriate prescription of antimicrobials.

"Okay, it's about the patient preferences, okay, it's just like there are a lot of antibiotics but 164 patients may choose that "I like this antibiotic when I take it I feel good, I recover from my 165 complaints and my disease”, could be just patient expectation according to clinical condition of 166 her disease” (Clinician \# 5).

167 "aah sometimes aah patient do have their preferences on which drugs antibiotics to be 168 prescribed to them that they feel is best for them not the condition they have, what's best for 169 them" (Clinician \# 6).

\section{Theme 2: Belief about Efficacy}

171 Most patients believe that intramuscular injections, IM and IV antibiotics work better than per os 172 (PO), so, if you give them PO antibiotics, they believe that you have not helped them. They 173 believe that if you give them antibiotics they will dramatically change for the better within two 174 days.

175 'Patients' understanding of antimicrobials is that the belief that antimicrobials, especially 176 injectable heal any form of severe illness. Even if it is not a bacterial infection, they still think 177 that if you give them IV [Intravenous] antimicrobials, they are going to recover" (Clinician \# 7).

178 Clinicians also reported that prescribing antibiotics occurs even in suspected cases of viral 179 infections or mere cough because patients and guardians believe their patients will improve only 
180 after taking antibiotics and that any form of illness can be cured with antibiotics. Thus, patients

181 show a lack of knowledge on antimicrobials that can be used to treat a bacterial infection or a

182 viral infection.

183 "I think they feel that for them to get well then they have to take a certain type of antimicrobial.

184 Whether you find that the malaria test is negative then they still have the feeling that for them to 185 get well they have to take antimalarial, or for them they just have a viral infection like cough or 186 whatever or just a flu. They believe that for them to get well they have to take antibiotic like 187 amoxicillin" (Clinicians\# 12).

188 Clinicians reported that patients believe that having a cough means one needs antibiotics. This 189 belief prevents proper antimicrobial prescription in a health care setting.

Theme 3: Negative attitude of patients towards clinicians who do not prescribe 191 antimicrobials

192 Most of the clinicians reported that when they refuse to prescribe antimicrobials, patients think 193 that they do not know their work; they are sometimes referred to as not being good doctors 194 because of denying them what they want. In other words, the patients have a negative attitude 195 towards such clinicians.

196 "Well, when you refuse they think maybe you are not a good clinician. You don't really know 197 your work or else you haven't helped them. They would prefer to go to another clinician or else 198 to go to another hospital where they feel they can be helped. They feel that you haven't helped 199 them" (clinician \#12).

200 Almost all clinicians reported that such patients think of them as being incompetent when they 201 refuse to prescribe antimicrobials to them. What the patients do in such circumstances is to go to 202 another clinician. 
"They do just think that am not a medical practitioner, that am not well equipped with knowledge and they go for another clinician" (Clinician \# 4).

\section{Theme 4: Educating the patients}

As one way of reducing inappropriate antimicrobial prescription, the majority of Clinicians reported the need for patients' education during patients' clinic visits. Clinicians suggested that the following should be done during clinician-patient interaction in health care settings: educating patients on the dosage, how to take the drugs and for how long as well as any side effects. This needs to be properly communicated in addition to when to return to the hospital if there is no improvement.

"First, we need to explain why we are giving those drugs, frequency, route, and duration. And they should not share with someone else because it's only for him or them that have attended the service and the drug is prescribed only for him. I think about the problems people still share drugs somewhere behind. You should build a good relationship between you and the patient and make sure when you have given the drug they should come back for feedback" (Clinician \#11).

Patient education is a factor that can lead to a reduction in unnecessary antimicrobial prescriptions. This important theme of educating the patients is however restricted by the reality on the ground as shown in the next theme.

\section{Theme 5: Limited time /Clinicians being overwhelmed}

Clinicians stated that limited consultation time between clinician and patient was one of the factors that lead to inappropriate prescription of antimicrobial. It emerged that there is limited time a clinician can spend with an individual patient because of large numbers of patients visiting a health care setting which is a big challenge that affects clinicians. There is a lack of comprehensive history taking on patients because of having to spend minimal time with each one of them. Some clinicians may see as many as 50 patients per day. 
"We can spend 2 minutes with each patient because we have long lines in outpatient 228 departments and sometimes there is one clinician or two, so if you take much time with patients, 229 they start complaining that you are wasting their time. It affects a lot because we need to have 230 more time with our patient and they should talk more of their complaints but with the complaints 231 that I said that we are few Clinicians, we spend aah not enough time with the patient, so the 232 patient do not share more of their complaints that they have come with on that particular day" 233 (Clinician \#1).

234 The underlying factor is handling a long queue of patients in outpatient departments and huge 235 workload. This results in unnecessary antimicrobials prescription in health care settings in order 236 to relieve pressure in the outpatient department.

237 Lack of enough time is a barrier for proper history taking, physical examinations, investigations 238 and counselling. Furthermore, because of having fewer clinicians, antimicrobials are being 239 prescribed in order to see more patients within a short period of time and this results in no 240 explanation to patients on what they are suffering from and the importance of adhering to 241 medications.

\section{Theme 6: Hindrance / Obstacle to antimicrobial prescription}

243 The study also investigated problems of antimicrobial prescription in cases where this is the 244 appropriate decision. Clinicians stated that they do face problems and challenges in prescribing 245 some antimicrobials because of their unavailability in the health care settings. The findings 246 revealed that frequent unavailability, shortages and antibiotics being sold on the open market are 247 problems since people can go and buy without a prescription from a clinician.

248 "One of the challenges mostly (silence) its aah repetitive usage of single antimicrobial, even in 249 the same patients or in most outpatients seen or even inpatients, so it's mostly certain antibiotic 250 dominate over other antibiotics, so that's one of the challenges simply because it has developed 251 some resistance simply because of overuse and it has caused most of the unfavorable side effects 
252 and which are most difficult to treat so are some of the challenges we have met so far" 253 (Clinician \# 7).

254 Clinicians reported that a shortage of certain antimicrobials makes them prescribe the same 255 antibiotics, even in cases where they believe it is not the best option, not indicated or not the 256 strongest one.

257 "Your choices may be out of stock in a particular pharmacy and that can affect your prescription 258 as well. And the other thing is, you are not quite sure what you are treating. So you just 259 prescribe but then you are not really sure like blinded and treating blindly” (Clinician \# 12).

\section{Theme 7: Clinician Lack of Knowledge on antibiotic and antimicrobial resistance}

261 Besides all that, participants showed that they had minimal understanding of antibiotics and 262 antibiotic resistance. Four clinicians correctly defined what antibiotic and antimicrobial 263 resistance is, which represents $13 \% .6 .5 \%$ correctly defined antibiotic resistance whilst only $3 \%$ 264 correctly defined antimicrobial resistance. Clinicians were also using antibiotics and 265 antimicrobial resistance interchangeably. In this study, clinicians did not define properly what 266 antibiotics are and what antimicrobials are. Below is an illustration.

267 Definition of Antimicrobial resistance: Antimicrobial resistance means the causative organisms, 268 the bacteria have developed a mechanism or a resistance to that antimicrobial which means you 269 might give antimicrobial which previously could work or the bacteria could respond or could be 270 sensitive to that antimicrobial but now in the later stage or after a certain period of time the 271 bacteria will develop another mechanism against that antimicrobial” (Clinician \# 7).

\section{Discussion}

273 Medical Assistants, Clinical Technicians and Clinical officers are an important group in 274 prescribing antimicrobials in Sub Saharan African countries including Malawi. They are 275 frontline health workers in primary and secondary health facilities in Malawi. They are more 
276 likely to prevent inappropriate prescription of antimicrobials and educate patients if they have

277 enough knowledge and are aware of antimicrobial resistance. This study yields important

278 findings regarding factors influencing clinicians to give over their prescribing decision power to

279 patients during consultation. The allocation of more health workers and providing patient

280 education during consultation is crucial and paramount to improve antimicrobial prescription.

281 The study identifies an important area that needs to be addressed when developing education

282 interventions regarding interactions between clinician and patients. The study has demonstrated

283 that only few clinicians were aware on the definition of antibiotic and antimicrobial resistance.

284 The majority asserted that some factors influence clinicians to give over their prescribing

285 decision power to patients. Key among the factors are preferences, beliefs and efficacy of 286 antimicrobials, negative attitude of patients towards clinicians, limited time /Clinician being 287 overwhelmed as well as hindrance / obstacle to antimicrobial prescription.

288 However there is a significant gap on the definition of antibiotic and antimicrobial resistance 289 among Clinicians which needs to be bridged, and that can result into appropriate antimicrobial 290 prescription.

\section{Preference}

292 The present study confirmed that patient preference is a factor that influences clinicians to give 293 over their prescribing decision power to patients and it determines inappropriate antimicrobial 294 prescription in health care settings. These findings are supported by several studies in developed 295 countries [11,25-28]. In the USA, a similar study to the current one found out that parental 296 pressure was influencing clinicians to prescribe antibiotics [12]. This is also reflected in a similar 297 study, in which, one of the reasons for the prescription of antimicrobials is patient demands or 
298 attitude [29]. A study done in Egypt also reported that preferences of caregivers and patients

299 were among of the factors that contribute to antibiotics prescriptions [28].

300

301

302

303

304

305

306

307

308

309

310

311

312

313

314

315

316

317

318

319

\section{Belief about efficacy}

Our study also found out that belief about efficacy among patients in antimicrobials is contributing to inappropriate prescriptions in health care settings. These findings corroborate the findings from other studies.

It is reported that patients have a belief in certain antimicrobials over others when they visit health care settings. Clinicians in this study cited that patients' demands and preferences for injectable or intravenous antimicrobials over oral ones contribute to inappropriate prescription.

Similar findings from developed countries also reported that patients believe in antimicrobials when they visit health care settings, and even when they have a viral infection, they will demand antibiotics to avoid repeated consultations [30-33].

The current findings are also supported by another study which found out that patients come to a hospital with common cold and then demand intravenous antibiotics [34]. The current study findings also corroborate other studies which reported that there is belief that intravascular antibiotics are better than oral antibiotics and that, both doctors and patients encourage prescription of intravenous antibiotics [28,29,33-35].

\section{Negative attitude of patients towards clinicians}

Our findings about negative attitude of patients towards clinicians who refuse to prescribe antimicrobials is similar to other study findings which found out that clinicians were prescribing antibiotics in fear of losing patients' trust [36]. The results also show that patients would change physicians when antibiotics are not prescribed. This is also reflected in similar studies that 
320 reported that even when patients do not need medication, doctors prescribe antibiotics to

321 maintain a good patient-doctor relationship [37-39].

322 A study done in Malaysia found out that a few participants indicated that they would make their

323 expectations explicit and request antibiotics from their physician even when they had viral

324 infections, as they believe the medicine promotes rapid recovery. They also said they would

325 consult another physician if their request was not granted [40].

\section{Educating patients}

327 This study also revealed that clinicians were influenced to prescribe antimicrobials because of 328 patients' lack of education on medications. In general, the health systems in Malawi are 329 considered inadequate to meet the ever increasing health demanding population in health care 330 settings as health care and clinician-patient ratios still need to improve. Clinicians reported that 331 they do not have enough time to counsel and educate patients during consultations because of 332 demands on their time due to large patient numbers.

333 In a review conducted by Ayukekbong et al.[41], it was found out that, because of high patient334 doctor ratio in most developing countries, doctors are overwhelmed and, as a result, there is often 335 inadequate time for meaningful education and communication with the patient on drug adherence 336 guidelines and consequences of poor or non-adherence to the guidelines.

337 The current results corroborate a study done in North Carolina that revealed that clinicians 338 should provide information in a manner that is easy for patients to understand as to why an 339 antibiotic is not needed to treat a particular illness as well as how to appropriately use antibiotics 340 in their treatment as and when they are prescribed [42]. 
341 Providing education at all levels, that is, community, healthcare setting and individual, is

342 essential to ensuring rational use of antibiotics and suppressing inappropriate use.

343 Public education campaigns are effective in changing attitudes and knowledge regarding 344 antibiotic use and resistance. Fletcher-Lartey and Machowska [22,39] found out that consumer 345 education, such as discussion and explanation, was the common strategy reported by participants 346 to manage patients' expectations and demand for antibiotics.

347 Findings in this study are all consistent with other studies done in Belgium, England and France 348 which reported that mass media interventions such as national TV campaigns and campaigns 349 through other forms of mass media have been shown to reduce antibiotic prescribing for Acute 350 Respiratory Tract Infection but argued that this strategy works best when targeting both 351 healthcare professionals and the public [43]. It is recommended that care providers, dispensers 352 and patients need to be educated on how the use and misuse of antimicrobials may contribute to 353 the development of resistance [41]. It is cited that lack of communication skills is a factor that 354 promotes unnecessary antimicrobial prescription [44].

\section{Limited -Time /Clinician being overwhelmed}

356 The findings also reveal that limited time act as a barrier to proper antimicrobial prescriptions.

357 Clinicians reported that they prescribe antimicrobials in order to handle long queues in the 358 outpatient department. Several studies support the fact that clinicians spend less time with 359 patients because of work overload.

360 It is reported that clinicians prescribe medications in order to end the consultation and the 361 clinicians themselves also reported that they prescribe under pressure when factors other than 
362 clinical presentation pushed them into prescribing even when they believe antibiotics are not 363 needed [32].

364 In a study conducted in Karnataka state in South India, physicians agreed that they have too 365 much work because of staff shortages and nearly half of them said that their patients ask them to 366 prescribe antibiotics [48].

367 In this current study, clinicians do not perceive that limited time and inadequate number of 368 clinicians is a facilitator for inappropriate prescription of antimicrobials.

\section{Hindrance / Obstacle to antimicrobial prescription}

371 Several factors exist such as unavailability of antimicrobials or their shortage, as well as

372 antibiotics being sold on the open markets all of which are barriers to proper antimicrobial

373 prescriptions since people can go and buy antimicrobials from pharmacies and open markets

374 without a prescription from a clinician. One of the barriers to appropriate antibiotic prescriptions

375 is inappropriate antibiotic use which has resulted from lack of access to and affordability of 376 antibiotics due to inadequate government funding in developing countries [50].

377 In another study, it is also reported that those who were on medical aid were more likely to 378 receive an antibiotic than those not on medical aid [46].

379 A study done by Baubie, et al. [51] also reported that high physician workload and high 380 antibiotic use in the community were major barriers to antimicrobial stewardship implementation 381 and lack of patient or client understanding of antibiotics, and difficulty in making diagnoses were 382 barriers to proper antimicrobial prescription [52]. 
383 The current study is also supported by another study done in India which shows that selection of

384 particular antibiotics also depends on their availability at the public health center and this is a 385 barrier to prescribers [48]. The above study findings also corroborate other results, which report 386 that clinicians felt that some antibiotics available in their hospital are of poor quality and less 387 effective or that the required ones are not available and the patient gets antibiotics directly from 388 shopkeepers without prescriptions [24]. Similarly, a study done in South Asia reveal that 389 common challenges to proper antimicrobials prescription were poor dispensing, poor quality 390 antibiotics, less effective ones in hospital, insufficient history taking and sale of antibiotics that 391 have no proper dosage or are clinically inappropriate [53].

392 In fact, a study done in India found out that one of the obstacles to the appropriate use of 393 antibiotics is poor quality of antibiotics and less effective ones in hospitals [24].

\section{Clinician Lack of Knowledge on antibiotic and antimicrobial resistance}

396 Furthermore, we need to educate clinicians on antibiotic and antimicrobial resistance. Overall, 397 participants had minimal understanding of antibiotics and antimicrobials resistance. In this study, 398 clinicians pointed out that overuse, poor adherence, and self-medication were causes of antibiotic 399 resistance. In a study done in France and Scotland, the clinicians had knowledge of antibiotics 400 resistance [54].

401 But, overall, in the current study, clinicians knew the causes of antimicrobial resistance and had 402 knowledge which is similar to the findings of a study done in Saud Arabia on rural and urban 403 physicians which pointed out that inadequate prescription, use of antimicrobials without 404 prescription and noncompliance of patients are the most important factors contributing to the 
405 development of bacterial resistance to antibiotics [54,55]. Studies done in Sudan and Ghana also

406 found out that a number of factors were mostly perceived by the majority of physicians as very

407 important causes of antibiotic resistance such as overuse in the population and hospitals, self-

408 medication, uncompleted antibiotics therapy, inappropriate empiric choice and low antibiotics

409 dosage use in animals as well [56,57].

410 Nicholson et al. [58] similarly reported that factors contributing to antibiotic resistance are: wide

411 spread use of antibiotics, overuse of a broad-spectrum of antibiotics, inappropriate use,

412 inadequate hand washing and use of antibiotics in animals.

413 In a similar study done in Ghana among prescribers, causes of antibiotics resistance identified

414 include antibiotics over-prescription, irrational prescription of antibiotics and patients'

415 noncompliance to medications [59].

\section{Strength and Limitation of the study}

417 This is the first study done in Malawi among clinical officers on antimicrobial prescription. The 418 study managed to capture a wide range of determinants of inappropriate antimicrobial

419 prescription. Sample size in qualitative research is determined by data saturation and it is a gold 420 standard in a qualitative study however $(n=30)$ were all interviewed. The study had high levels of 421 participation which might show that research participants were interested in antimicrobial 422 resistance and they were willing to participate in the study. This study was only done in one 423 district, Mulanje, Southern Malawi, so it is a snapshot of Mulanje district as such the result 424 cannot be generalized. Another limitation is non-random sampling. Finally, private clinicians 425 were not interviewed which is also one of the limitations of the study.

\section{Conclusion}


427 This study sought to assess determinants of antimicrobial prescription among clinicians in 428 Mulanje, Malawi. Based on the findings in this study, the following are key conclusions that 429 contribute to the evidence of determinants of antimicrobial prescription. This is one of the first 430 few studies in southern Malawi and will contribute to evidence based targeted interventions to 431 address the problem of inappropriate antimicrobial prescription in health care settings.

432 Although clinicians were aware of the causes of antimicrobial resistance, they showed lack of 433 knowledge on antimicrobial resistance. Lack of patient education, limited time and work 434 overload are among the factors that promote inappropriate prescription of antimicrobials.

\section{Supplementary information}

\section{Author Contributions:}

$439 \mathrm{MC}, \mathrm{CB}$ and FK wrote the Manuscript, MC, CB and FK design the research and finally MC, CB 440 and FK analyses the data.

\section{Acknowledgements}

443 The authors acknowledge support from NORERD and are much grateful to all participants who 444 took part to participate in the study, Director of Health and Social Services at Mulanje District 445 Hospital and Director of Mulanje Mission Hospital for allowing us to collect data, Daniel 446 Nyanjagha and Ron Nserebo for supporting the study during the whole academic period.

449 The authors report no conflicts of interest in this work. 
450 Ethics approval Ethics

451 Ethics approval was obtained from the Malawi College of Medicine Research Ethics Committee

452 under approval number (ID P.04/19/2656).

453

$454 \quad$ Availability of data and materials

455 The datasets used and analyzed during the current study are available from the corresponding

456 author on reasonable request.

457 Consent for publication

$458 \quad$ Not applicable

459 Abbreviations

460 AMR: Antimicrobial resistant; ABR: Antibiotic resistant; LMICs: Low Middle Income

461 Countries; BRICS: Brazil, Russia, India, China and South Africa, SDM: Shared Decision

462 Making.

463

464

465

466

467

468

469

470

471

472 


\section{References}

474 1. Van Der Velden AW, Pijpers EJ, Kuyvenhoven MM, Tonkin-Crine SKG, Little P, Verheij TJM. Effectiveness of physician-targeted interventions to improve antibiotic use for respiratory tract infections. Br J Gen Pract. 2012;62(605):801-7.

2. Bai Y, Wang S, Yin X, Bai J, Gong Y, Lu Z. Factors associated with doctors' knowledge on antibiotic use in China. Sci Rep [Internet]. 2016;6:1-5. Available from: http://dx.doi.org/10.1038/srep23429

3. Surveillance A, Glass S. Fleming Fund AMR Surveillance in low- and settings A roadmap for participation in the global. 2016; Google Search [Internet].[cited 2019, January 13]

4. Musicha P, Cornick JE, Bar-Zeev N, French N, Masesa C, Denis B, et al. Trends in Available from:

https://wellcomeopenresearch.s3.amazonaws.com/supplementary/12527/99f63366743d473c-b3ad-e96403e4ab3e.pdf antimicrobial resistance in bloodstream infection isolates at a large urban hospital in Malawi (1998-2016): a surveillance study. Lancet Infect Dis [Internet]. 2017;17(10):1042-52. Available from: http://dx.doi.org/10.1016/S1473-3099(17)30394-8

5. Tam PYI, Musicha P, Kawaza K, Cornick J, Denis B, Freyne B, et al. Emerging resistance to empiric antimicrobial regimens for pediatric bloodstream infections in Malawi (19982017). Clin Infect Dis. 2019;69(1):61-8.

6. Mangione. How to prescribe few Antibiotics. 2019;94(3):200-2. GoogleSearch [Internet]. 
495 7. Jiskoot P. On-the-job training of clinical officers in Malawi. 2008;20(September):74-7.

496 Google Search[internet].[cited 2021 January 15] Available

497 from.https://www.ncbi.nlm.nih.gov/pmc/articles/PMC3345688/.

498 8. Mendelson M, Dar OA, Hoffman SJ, Laxminarayan R, Mpundu MM, Røttingen JA. A

499 Global Antimicrobial Conservation Fund for Low- and Middle-Income Countries. Int J

$500 \quad$ Infect Dis. 2016;51:70-2.

501 9. Eibs T, Koscalova A, Nair M, Grohma P, Kohler G, Bakhit RG, et al. Qualitative study of 502 antibiotic prescription patterns and associated Bissau, drivers in Sudan, Guinea- - Central 503 African Republic and Democratic Republic of Congo. 2020;1-12.

504 10. Tadesse BT, Ashley EA, Ongarello S, Havumaki J, Wijegoonewardena M, González IJ, et 505 al. Antimicrobial resistance in Africa: A systematic review. BMC Infect Dis. $506 \quad 2017 ; 17(1): 1-17$.

507 11. Lum EPM, Page K, Whitty JA, Doust J, Graves N. Antibiotic prescribing in primary 508 healthcare: Dominant factors and trade-offs in decision-making. Infect Dis Heal [Internet].

$509 \quad$ 2018;23(2):74-86. Available from: https://doi.org/10.1016/j.idh.2017.12.002

510 12. Szymczak JE, Feemster KA, Zaoutis TE, Gerber JS. Pediatrician Perceptions of an 511 Outpatient Antimicrobial Stewardship Intervention. Infect Control Hosp Epidemiol. $512 \quad 2014 ; 35(\mathrm{~S} 3):$ S69-78.

513 13. Brookes-Howell L, Hood K, Cooper L, Coenen S, Little P, Verheij T, et al. Clinical 514 influences on antibiotic prescribing decisions for lower respiratory tract infection: A nine 515 country qualitative study of variation in care. BMJ Open. 2012;2(3):e000795. 
516 14. Pinder R, Sallis A, Berry D, Chadborn T. Behaviour change and antibiotic prescribing in 517 healthcare settings Literature review and behavioural analysis About Public Health 518 England About the Department of Health. 2015;(June).

519 15. Teixeira Rodrigues A, Roque F, Falcão A, Figueiras A, Herdeiro MT. Understanding 520 physician antibiotic prescribing behaviour: A systematic review of qualitative studies. Int $521 \quad$ J Antimicrob Agents. 2013;41(3):203-12.

522 16. Hruza H, Velasquez T, Madaras-Kelly K, Fleming-Dutra K, Samore M, Butler J. 1888.

523 Clinicians' Beliefs, Knowledge, Attitudes, and Planned Behaviors on Antibiotic

524

525

526

527

17. Chandra RV, C N A, Kj N, Dhamodaran T. Doctor - Patient Relationship - a Review. $2013 ; 11-3$.

18. Haque M, Rahman NIA, Zulkifli Z, Ismail S. Antibiotic prescribing and resistance:

Knowledge level of medical students of clinical years of university Sultan Zainal Abidin, Malaysia. Ther Clin Risk Manag. 2016;12:413-26.

19. Llor C, Bjerrum L. Antimicrobial resistance: Risk associated with antibiotic overuse and 532 initiatives to reduce the problem. Ther Adv Drug Saf. 2014;5(6):229-41.

20. Anderson T. Antimicrobial stewardship in Australian health care. Vol. 1, JACAntimicrobial Resistance. 2019. 12 p.

21. Manafa O, McAuliffe E, Maseko F, Bowie C, MacLachlan M, Normand C. Retention of 536 health workers in Malawi: Perspectives of health workers and district management. Hum 
Resour Health. 2009;7:1-9.

538 22. Fletcher-Lartey S, Yee M, Gaarslev C, Khan R. Why do general practitioners prescribe antibiotics for upper respiratory tract infections to meet patient expectations: A mixed methods study. BMJ Open. 2016;6(10):1-8.

541 23. Hawkings NJ, Wood F, Butler CC. Public attitudes towards bacterial resistance: A 542 qualitative study. J Antimicrob Chemother. 2007;59(6):1155-60.

543 24. Ghosh A, Deb T, Ghosh S. Knowledge, attitudes and practice survey about antimicrobial 544 545

26. Davari M, Khorasani E, Tigabu BM. Factors Influencing Prescribing Decisions of 550 Physicians: A Review. Ethiop J Health Sci. 2018;28(6):795-804.

27. Sturrock A, Landes D, Robson T, Bird L, Ojelabi A, Ling J. An audit of antimicrobial prescribing by dental practitioners in the north east of England and Cumbria. BMC Oral Health. 2018;18(1):1-8.

28. Kandeel A, El-Shoubary W, Hicks LA, Fattah MA, Dooling KL, Lohiniva AL, et al. Patient attitudes and beliefs and provider practices regarding antibiotic use for acute respiratory tract infections in minya, egypt. Antibiotics. 2014;3(4):632-44. 
Wards of a Tertiary Care Hospital in Malaysia. Int J Sci Environ Technol [Internet]. 2012;1(4):274-84. Available from: http://www.ijset.net/journal/40.pdf

560

561

562

563

564

565

566

567

568

569

570

571

572

573

574

575

576

577

578

30. Cals JWL, Boumans D, Lardinois RJM, Gonzales R, Hopstaken RM, Butler CC, et al. Public beliefs on antibiotics and respiratory tract infections: An internet-based questionnaire study. Br J Gen Pract. 2007;57(545):942-7.

31. Gaarslev C, Yee M, Chan G, Fletcher-Lartey S, Khan R. A mixed methods study to understand patient expectations for antibiotics for an upper respiratory tract infection. Antimicrob Resist Infect Control [Internet]. 2016;5(1):1-9. Available from: http://dx.doi.org/10.1186/s13756-016-0134-3

32. Lucas PJ, Cabral C, Hay AD, Horwood J. A systematic review of parent and clinician views and perceptions that influence prescribing decisions in relation to acute childhood infections in primary care. Scand J Prim Health Care. 2015;33(1):11-20.

33. Roope LSJ, Tonkin-Crine S, Butler CC, Crook D, Peto T, Peters M, et al. Reducing demand for antibiotic prescriptions: Evidence from an online survey of the general public on the interaction between preferences, beliefs and information, United Kingdom, 2015. Eurosurveillance. 2018;23(25):1-11.

34. Broom J, Broom A, Adams K, Plage S. What prevents the intravenous to oral antibiotic switch? A qualitative study of hospital doctors' accounts of what influences their clinical practice. J Antimicrob Chemother. 2016;71(8):2295-9.

35. Prajapati V, Bhatt JD, Vora MB. Study Of Prescribing Patterns Of Antimicrobial Agents In The Medicine Department At Tertiary Teaching Care Hospital In Gujarat. 
36. Saha SK. Factors Influencing Clinician's Antibiotic Prescribing Behaviors (APB) in Bangladesh: An In-Depth Review Using COMB Model. Open Access J Transl Med Res. 2017;1(4):91-5.

37. El Sherbiny NA, Ibrahim EH, Masoud M. Assessment of knowledge, attitude and behavior towards antibiotic use in primary health care patients in Fayoum Governorate, Egypt. Alexandria J Med [Internet]. 2018;54(4):535-40. Available from: https://doi.org/10.1016/j.ajme.2018.06.001

38. Lee TH, Wong JGX, Lye DCB, Chen MIC, Loh VWK, Leo YS, et al. Medical and psychosocial factors associated with antibiotic prescribing in primary care: Survey questionnaire and factor analysis. Br J Gen Pract. 2017;67(656):e168-77.

39. Machowska A, Lundborg CS. Drivers of irrational use of antibiotics in Europe. Int J Environ Res Public Health. 2019;16(1).

40. Irawati L, Alrasheedy AA, Hassali MA, Saleem F. Low-income community knowledge, attitudes and perceptions regarding antibiotics and antibiotic resistance in Jelutong District, Penang, Malaysia: A qualitative study. BMC Public Health. 2019;19(1):1-15.

41. Ayukekbong JA, Ntemgwa M, Atabe AN. The threat of antimicrobial resistance in developing countries: Causes and control strategies. Vol. 6, Antimicrobial Resistance and Infection Control. 2017.

42. Davis ME, Liu TL, Taylor YJ, Davidson L, Schmid M, Yates T, et al. Exploring patient awareness and perceptions of the appropriate use of antibiotics: A mixed-methods study. 

Available

602

603

604

605

606

607

608

609

610

611

612

613

614

615

616

617

618

619

from. https://www.ncbi.nlm.nih.gov/pmc/articles/PMC5745466/.

43. O’Connor R, O’Doherty J, O’Regan A, Dunne C. Antibiotic use for acute respiratory tract infections (ARTI) in primary care; what factors affect prescribing and why is it important? A narrative review. Ir J Med Sci. 2018;187(4):969-86.

44. Teng CL. Antibiotic prescribing for upper respiratory tract infections in the Asia-Pacific region: A brief review. Malaysian Fam Physician. 2014;9(2):18-25.

45. Meropol SB, Votruba ME. Decision-making and the barriers to judicious antibiotic use. Pediatrics. 2015;136(2):387-8.

47. Jeffrey A. Linder. HHS Public Access. Physiol Behav. 2018;176(1):139-48.

48. Krishna1 S, Erica Vlieghe, N. S. Prashanth, Bhanu Prakash, Asm Shahabuddin VR. Perception of health-care staff's knowledge, attitudes, and practices and of potential barriers toward containing antibiotic resistance in public health-care facilities of Tumkur district (South India). Ann Trop Med Public Heal. 2017;10(6):1587-90.

50. Frost I, Craig J, Joshi J, Faure K, Laxminarayan R. Access Barriers to Antibiotics. Washington, DC Cent Dis Dyn Econ Policy. 2019;26.

51. Baubie K, Shaughnessy C, Kostiuk L, Varsha Joseph M, Safdar N, Singh SK, et al. Evaluating antibiotic stewardship in a tertiary care hospital in Kerala, India: A qualitative interview study. BMJ Open. 2019;9(5):1-7. 
620 52. Zhuo A, Labbate M, Norris JM, Gilbert GL, Ward MP, Bajorek B V., et al. Opportunities

621

622

623

624

625

626

627

628

629

630

631

632

633

634

635

636

637

638

639

640

and challenges to improving antibiotic prescribing practices through a One Health approach: Results of a comparative survey of doctors, dentists and veterinarians in Australia. BMJ Open. 2018;8(3):1-12.

53. Tangcharoensathien V, Chanvatik S, Sommanustweechai A. Complex determinants of inappropriate use of antibiotics. Bull World Health Organ. 2018;96(2):141-4.

54. Pulcini C, Williams F, Molinari N, Davey P, Nathwani D. Junior doctors' knowledge and perceptions of antibiotic resistance and prescribing: A survey in France and Scotland. Clin Microbiol Infect [Internet]. 2011;17(1):80-7. Available from:

http://dx.doi.org/10.1111/j.1469-0691.2010.03179.x

55. Al-Homaidan HT, Barrimah IE. Physicians' knowledge, expectations, and practice regarding antibiotic use in primary health care. Int J Health Sci (Qassim) [Internet]. 2018;12(3):18-24. Available from:

http://www.ncbi.nlm.nih.gov/pubmed/29896067\%0Ahttp://www.pubmedcentral.nih.gov/a rticlerender.fcgi?artid=PMC5969788

56. Kheder S. Physicians' Knowledge and Perception of Antimicrobial Resistance: A Survey in Khartoum State Hospital Settings. Br J Pharm Res. 2013;3(3):347-62.

57. Labi AK, Obeng-Nkrumah N, Bjerrum S, Aryee NAA, Ofori-Adjei YA, Yawson AE, et al. Physicians' knowledge, attitudes, and perceptions concerning antibiotic resistance: A survey in a Ghanaian tertiary care hospital. BMC Health Serv Res. 2018;18(1):1-12.

58. Nicholson A, Tennant I, White L, Thoms-Rodriguez CA, Cook L, Johnson S, et al. The 
641

642

643 59. Asante KP, Boamah EA, Abdulai MA, Buabeng KO, Mahama E, Dzabeng F, et al.

644

645

646

647

648

knowledge, attitudes and practices of doctors regarding antibiotic resistance at a tertiary care institution in the Caribbean. Antimicrob Resist Infect Control. 2018;7(1):1-9. Knowledge of antibiotic resistance and antibiotic prescription practices among prescribers in the Brong Ahafo Region of Ghana; A cross-sectional study. BMC Health Serv Res. 2017;17(1):1-9. 